\title{
Evaluation of a 3D surface imaging system for deep inspiration breath-hold patient positioning and intra-fraction monitoring
}

\author{
Vincent C. Hamming ${ }^{1 *}$ (D), Christa Visser ${ }^{1}$, Estelle Batin ${ }^{1}$, Leah N. McDermott², Dianne M. Busz ${ }^{1}$, Stefan Both', \\ Johannes A. Langendijk ${ }^{1}$ and Nanna M. Sijtsema ${ }^{1}$
}

\begin{abstract}
Purpose: To determine the accuracy of a surface guided radiotherapy (SGRT) system for positioning of breast cancer patients in breath-hold $(\mathrm{BH})$ with respect to cone-beam computed tomography (CBCT). Secondly, to evaluate the thorax position stability during BHs with SGRT, when using an air-volume guidance system.

Methods and materials: Eighteen left-sided breast cancer patients were monitored with SGRT during CBCT and treatment, both in $\mathrm{BH}$. CBCT scans were matched on the target volume and the patient surface. The setup error differences were evaluated, including with linear regression analysis. The intra-fraction variability and stability of the airvolume guided BHs were determined from SGRT measurements. The variability was determined from the maximum difference between the different $\mathrm{BH}$ levels within one treatment fraction. The stability was determined from the difference between the start and end position of each $\mathrm{BH}$.

Results: SGRT data correlated well with CBCT data. The correlation was stronger for surface-to-CBCT (0.61) than target volume-to-CBCT (0.44) matches. Systematic and random setup error differences were $\leq 2 \mathrm{~mm}$ in all directions. The $95 \%$ limits of agreement (mean \pm 2 SD) were $0.1 \pm 3.0,0.6 \pm 4.1$ and $0.4 \pm 3.4 \mathrm{~mm}$ in the three orthogonal directions, for the surface-to-CBCT matches. For air-volume guided BHs, the variability detected with SGRT was $2.2,2.8$ and $2.3 \mathrm{~mm}$, and the stability - 1.0, 2.1 and $1.5 \mathrm{~mm}$, in three orthogonal directions. Furthermore, the SGRT system could detect unexpected patient movement, undetectable by the air-volume BH system.

Conclusion: With SGRT, left-sided breast cancer patients can be positioned and monitored continuously to maintain position errors within $5 \mathrm{~mm}$. Low intra-fraction variability and good stability can be achieved with the air-volume BH system, however, additional patient position information is available with SGRT, that cannot be detected with air-volume BH systems.
\end{abstract}

Keywords: Surface guided radiotherapy, Deep inspiration breath-hold, Breast cancer, Cone-beam computed tomography

\section{Summary}

In this study, the accuracy of a 3D surface guided radiotherapy (SGRT) imaging system was evaluated for the positioning of deep inspiration breath-hold radiotherapy breast cancer patients. Furthermore, the SGRT system was used to evaluate the patient surface stability when breathholds were guided by an air-volume monitoring system.

\footnotetext{
* Correspondence: v.c.hamming@umcg.nl

'Department of Radiation Oncology, University of Groningen, University Medical Center Groningen, Hanzeplein 1, 9713, GZ, Groningen, The Netherlands

Full list of author information is available at the end of the article
}

Promising results indicated that the SGRT is accurate with respect to cone-beam $\mathrm{CT}$, and can reliably monitor the thorax position of patients undergoing deep inspiration breath-hold radiotherapy.

\section{Introduction}

Respiratory motion introduces a variable distance between the high-dose target and the heart for left-sided breast cancer patients. Since the heart is in close proximity to the target volume, these patients are at risk of radiationinduced cardiac toxicity [1]. Deep inspiration breath-hold $(\mathrm{DIBH})$ is a technique used to increase the separation 
between the heart and the target, and eliminate the influence of breathing motion [2-5]. However, the inter- and intra-fraction variability of the breath-hold $(\mathrm{BH})$ position can compromise the benefits of DIBH [6].

The active breathing coordinator $(\mathrm{ABC}, \mathrm{R} 3.0$, Elekta $A B$, Stockholm, Sweden) is a commercially available system designed to guide patient's $\mathrm{BHs}$ [7]. The $\mathrm{ABC}$ system solely supports a reproducible breathing airvolume per $\mathrm{BH}$ however it does not verify the thorax position. Patients can employ abdominal or thoracic breathing, and non-breathing related movement is possible, while expelling consistent volumes of air [8]. Therefore, the actual position of the thorax could still vary, while using the $A B C$ system.

Prior to irradiation, the $\mathrm{BH}$ position can be verified by imaging. Generally images from either electronic portal imaging devices (EPID, 2D) or cone-beam computed tomography (CBCT, 2D or 3D) are used to verify the position of the thorax [9]. However, these modalities deliver additional radiation to the patient and are therefore suboptimal for intra-fraction monitoring of the $\mathrm{BH}$ position.

A solution to this problem is provided by surface-guided radiation therapy (SGRT). SGRT uses (non-invasive) optical surface imaging to reduce localization uncertainty during irradiation. This is achieved by continuously monitoring the patient's surface during treatment and comparing it to a reference position [10]. Using SGRT during treatment allows for less patient fixation and greater speed of setup which results in increased patient comfort [10-12]. Studies have shown that SGRT can potentially replace the use of skin markers for positioning $[13,14]$. However, large positioning differences can still remain between the patients' external surface and the internal structures on which radiotherapy treatment plans are based [15].

The goal of this study is two-fold: first, to determine the accuracy of a surface imaging system AlignRT (Version 5.0.1749, Vision RT Ltd., London, UK) for position verification of breast cancer patients in $\mathrm{BH}$ compared to CBCT data, registered to either the clinical target volume or to a section of the patient's surface. Secondly, to evaluate the intra-fraction variability and stability of the thorax position in $\mathrm{BHs}$ guided with the $\mathrm{ABC}$ system for breast cancer patients using AlignRT.

\section{Methods}

\section{Patient data}

Eighteen consecutive left-sided breast cancer patients were included in this study. In total, 16 patients received whole breast radiotherapy (WBRT), one patient received WBRT including the axilla and one patient received WBRT including a simultaneous integrated boost. All patients received DIBH treatments. Ten patients were treated with partial volumetric modulated arc therapy (par-VMAT) while eight patients were treated with conformal tangential fields. The parVMAT technique consisted of $70 \%$ tangential open fields and a 30\% VMAT contribution to optimize a homogenous dose distribution. All treatment plans were robustly planned, which was achieved by using a (skin) flash of $5 \mathrm{~mm}$ during optimization, to account for intra- or inter-fractional target motion [16]. One patient changed to free breathing (FB) radiotherapy after 5 fractions due to difficulties in maintaining BHs. For one patient, 5 treatment fractions were excluded from the analysis due to a broken mouthpiece.

\section{Breath-hold guidance}

The $\mathrm{ABC}$ device was used to guide breath-holds. It consists of a mouthpiece connected to a spirometer (to measure air-flow) and is coupled to a balloon valve [7]. The nose is pegged to ensure any breathing passes only through the mouthpiece. All patients received $\mathrm{ABC}$ training prior to $\mathrm{CT}$ acquisition. The valve within the system is closed at $75 \%$ of the maximum inhalation volume, which was determined during training. All patients received visual feedback to help the patient achieve an adequate inhalation volume. By closing the valve, the patient experienced a forced $\mathrm{BH}$ (no inhale or exhale was possible).

\section{Imaging preparation CT acquisition}

All patients received an ABC-guided DIBH CT scan (Somatom-Definition AS, Siemens, Forchheim, Germany) for treatment planning preparation. The cranial-caudal range of the scan region was from the diaphragm to the mandible. The slice thickness for all CT acquisitions was $2.0 \mathrm{~mm}$, whereas the in-plane resolution was $1.0 \mathrm{~mm}$. All patients were scanned in the Head-First-Supine orientation. Patients were positioned with a breast-board (CIVCO Medical Solutions) where the ipsi-lateral arm was placed above the head and the contra-lateral arm was positioned on the treatment table alongside the body. The treatment reference point was marked with tattoos and radiopaque wires.

\section{Setup verification \& registration}

Patient positioning during treatment was initially performed using skin marks. Patients were shifted to the treatment isocenter with respect to the marked reference points. Pretreatment CBCTs (Elekta Infinity ${ }^{\mathrm{ma}}$ linear accelerator, (gantry range: $-15^{\circ}$ to $180^{\circ}, 120 \mathrm{kV}, 32.5 \mathrm{~s}, 0.4$ $\mathrm{mAs}$ ) were acquired in $\mathrm{BH}$ position during the first three fractions and then weekly, and were used for online position correction. Three patients received daily pretreatment CBCTs because of large variations in daily setup errors. The CBCT was acquired in two or three separate $\mathrm{BHs}$ as the acquisition time was too long for a single $\mathrm{BH}$. Registration of the CBCT to the planning CT was 
performed in two ways. The first registration was the clinical match, an automatic online registration to the thoracic wall. The surgical clips marking the surgical cavity should be within $5 \mathrm{~mm}$ of their planning CT position. If at least one clip was $>5 \mathrm{~mm}$, the patient was repositioned. This method ensures optimal target coverage while minimizing the heart dose. After the registration to the thoracic wall, any deviation between the $\mathrm{CBCT}$ and the planning $\mathrm{CT}$ at the breast surface is immediately visible. If the position of the surgical clips or the breast surface deviated from the planning CT by $>5 \mathrm{~mm}$ for $>2$ treatment fractions, a repeat $\mathrm{CT}$ scan was performed to evaluate the dose distribution and the treatment plan was adapted if necessary. Resulting setup corrections were applied in 3D prior to irradiation. The second method was a manual offline registration to a section of the patient's surface. A similar region of interest (ROI) was used for the CBCTplanning CT registration as the ROI defined in the AlignRT procedure (see below).

\section{Surface imaging \& registration}

AlignRT was installed in the treatment room with a threepod configuration. A RealTimeDelta tool, provided by VisionRT, stores the surface position deviation per frame in a text file for post-processing, for three orthogonal and three rotational degrees of freedom. A 3D body surface of the patient was generated by the planning system RayStation (Version 6.1.1.2, RaySearch, Sweden) based on the planning CT and exported to AlignRT. After importing the patient surface contour in AlignRT, the user defined a ROI for monitoring the patient's position. The differences in each of the 6 degrees of freedom are calculated within this user-defined ROI. A standard ROI was used for every patient, as shown in Fig. 1. This ROI includes the breast with an isotropic margin of a few centimeters to ensure proper visualization of the ROI and to optimize the positioning error calculated by AlignRT. Only the left breast was included in the ROI. This has been shown to be more accurate than monitoring both breasts [17]. By using a rigid registration algorithm, AlignRT computed the deviation between the ROI and the reference surface. An average deviation is displayed for each of the six degrees of freedom. AlignRT was only used to monitor the patient during $\mathrm{CBCT}$ acquisition and treatment. In this study, AlignRT generated patient positioning data, hence, use of AlignRT did not influence the treatment.

\section{Comparison and analysis}

The AlignRT RealTimeDelta text files were imported in Excel (v2010, Microsoft) and were analyzed using an in-house macro-program. Figure 2 shows two examples of the data exported from AlignRT. Timeresolved, translational setup errors during a $\mathrm{BH}$ were recorded, rotations were not included.

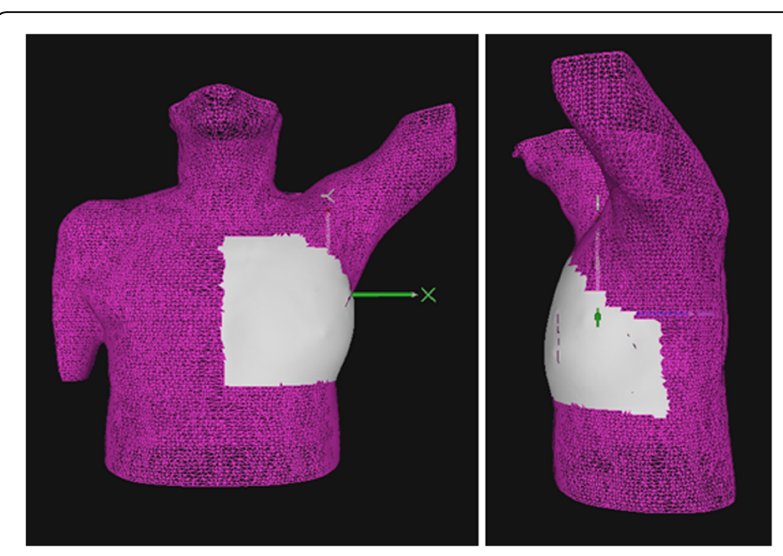

Fig. 1 The 3D body surface of the patient from the planning $C T$ is represented by a pink mesh. The region of interest (ROI) used for monitoring the patient surface is shown in grey

Moreover, the $\mathrm{BHs}$ performed during $\mathrm{CBCT}$ acquisition (setup analysis) were analyzed separately from the $\mathrm{BHs}$ performed during treatment (intra-fraction analysis). The differences between the setup errors determined from the $\mathrm{CBCT}$ and AlignRT data averaged over the duration of the $\mathrm{CBCT}$ acquisition were determined in the three main translational directions: left-right (LR), cranial-caudal (CC) and anterior-posterior (AP). The differences were calculated in terms of the group mean $(M)$, the systematic error $(\Sigma)$, the random error $(\sigma)$ and the $95 \%$ limits of agreement (LOA) according to the Bland-Altman analysis: $M \pm 1.96 \times S D$, with $M$ the mean and $S D$ the standard deviation over all fractions [18]. A linear regression analysis was performed and the values for the Pearson correlation coefficients $R$ were determined.

The AlignRT data during treatment was analyzed for the variability in intra-fraction position and the stability of $\mathrm{BHs}$ performed with the $\mathrm{ABC}$ system. The variability was determined from the maximum difference between the different $\mathrm{BH}$ levels within one treatment fraction for a patient. The stability was determined from the difference between the start and end position of each $\mathrm{BH}$.

\section{Results}

A total of 143 treatment fractions with online CBCTs for 18 patients were evaluated, with an average of $7.9 \pm 3.6$ CBCTs per patient. The CBCTs showed slight shading at the surface of the patient due to multiple BHs. No other artifacts in the CBCTs were observed. AlignRT was used to monitor the patient with 4-6 frames per second, depending on the size of the ROI used. The data showed no evidence of camera occlusion at any gantry angle.

Table 1 lists the $M, \Sigma, \sigma, R^{2}$ and LOA calculated for the differences in setup errors between AlignRT and CBCT target volume or surface registrations. The $\Sigma$ and $\sigma$ are equivalent or smaller for the $\mathrm{CBCT}$ surface registered results. 


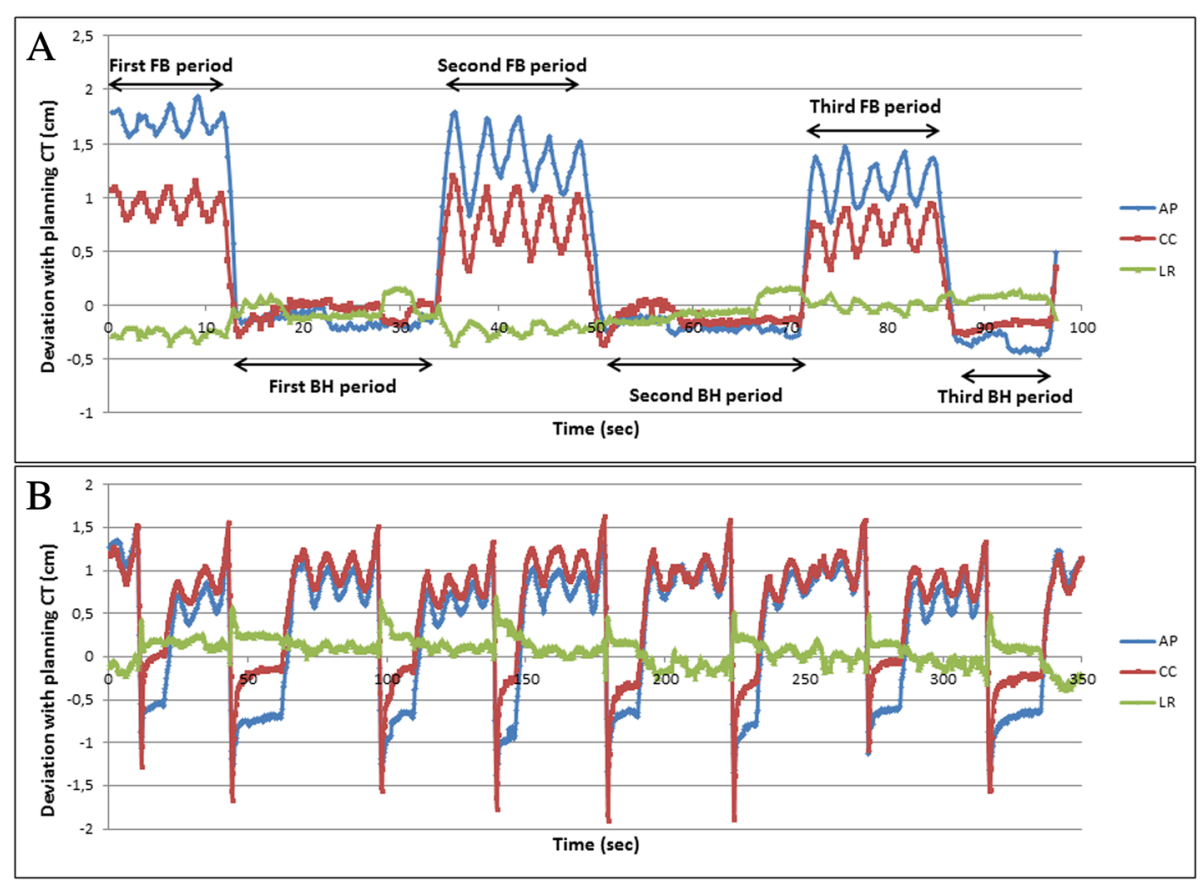

Fig. 2 a Clinical AlignRT data for a BH patient during CBCT acquisition. In this case three BHs were necessary for the scan. Only the BH periods were used for setup analysis. b Clinical AlignRT treatment data for the BH patient with the broken mouthpiece which clearly shows stability deviations $>14 \mathrm{~mm}$ in the $C C$ direction, while the air volume measured with the $A B C$ remained constant

Figure 3 shows the correlation between the AlignRT and CBCT setup errors. In Fig. 4 the Bland-Altman plots are presented for the setup errors of AlignRT and the CBCT registrations. LOA values are slightly closer when the $\mathrm{CBCT}$ is registered to the patient's surface than that of the target volume, however the difference between external (surface) and internal registration methods is small (mean difference is $1.3 \mathrm{~mm}$ ).

For the variability and stability of the treatment $\mathrm{BHs}$, a total of $1705 \mathrm{BHs}$ were analyzed in 261 treatment fractions. The results for the variability and stability are shown in Table 2 . The average variability was $2.4 \mathrm{~mm}$ and was similar in all directions. The maximum variability was $12.4 \mathrm{~mm}$ in the $\mathrm{CC}$ direction. The stability was negative in the LR direction and positive in the CC and AP direction. The absolute average stability was $1.5 \mathrm{~mm}$ and the maximum was 11.7 $\mathrm{mm}$. Figure $2 \mathrm{~b}$ shows sample data from one of the five excluded fractions with highly unstable BHs. During BH, the breast position increased in both the anterior and cranial directions due to a broken mouthpiece.

\section{Discussion}

Multiple studies have proven the high accuracy of AlignRT corrections with respect to 2D portal imaging [19-24]. In our study 3D CBCT imaging was used to verify AlignRT corrections, indicating suitability of using SGRT for intra-

Table 1 Overview of the setup differences between AlignRT and CBCT (target volume and surface registered)

\begin{tabular}{|c|c|c|c|c|c|c|}
\hline & \multicolumn{3}{|c|}{ AlignRT / CBCT target volume } & \multicolumn{3}{|c|}{ AlignRT / CBCT surface } \\
\hline & $\mathrm{LR}(\mathrm{mm})$ & $\mathrm{CC}(\mathrm{mm})$ & $\overline{A P}(\mathrm{~mm})$ & $\mathrm{LR}(\mathrm{mm})$ & $\mathrm{CC}(\mathrm{mm})$ & $\overline{\mathrm{AP}(\mathrm{mm})}$ \\
\hline$M$ & 0.1 & -0.5 & 1.7 & 0.1 & 0.6 & 0.4 \\
\hline$\Sigma$ & 1.8 & 1.4 & 1.9 & 1.0 & 1.4 & 1.5 \\
\hline$\sigma$ & 1.4 & 1.6 & 1.9 & 1.2 & 1.4 & 1.0 \\
\hline$M-1.96 \times S D$ & -4.4 & -4.3 & -3.3 & -2.8 & -3.5 & -3.0 \\
\hline$M+1.96 \times S D$ & 4.5 & 3.2 & 6.6 & 3.1 & 4.7 & 3.8 \\
\hline$R^{2}$ & 0.43 & 0.53 & 0.35 & 0.74 & 0.44 & 0.64 \\
\hline
\end{tabular}

$M$ Group mean, $\Sigma$ The systematic error, $\sigma$ The random error, $M \pm 1.96 \times S D$ The $95 \%$ limits of agreement, $R^{2}$ The Pearson correlation coefficient, $L R$ Left-right, $C C$ Cranial-caudal, AP Anterior-posterior 


\section{$\mathrm{CBCT}$ registered on patient's surface}
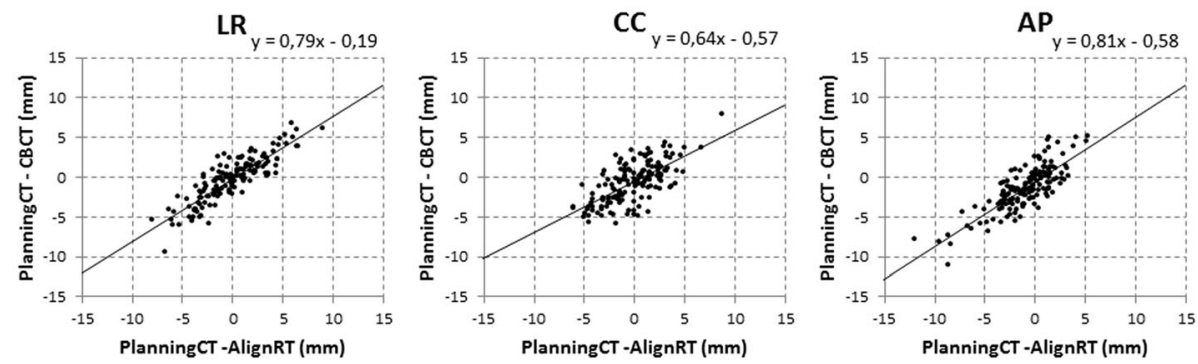

$\mathrm{CBCT}$ registered on target volume
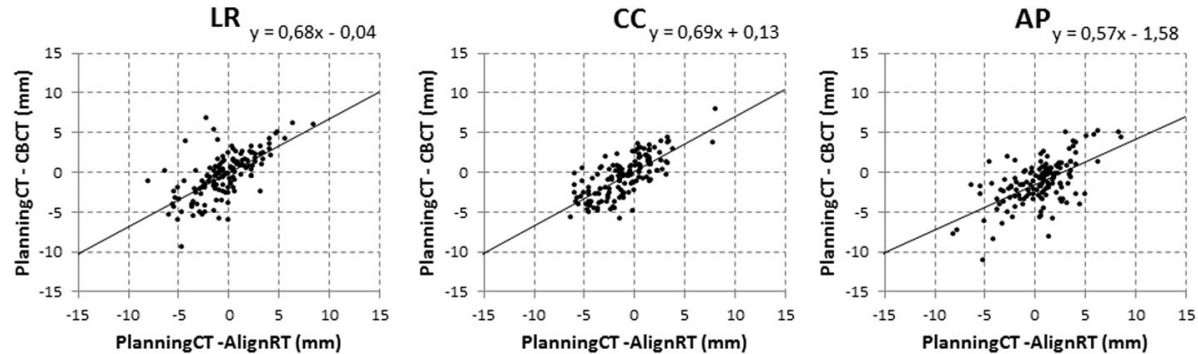

Fig. 3 Scatterplots with regression lines of the AlignRT setup errors vs the CBCT setup errors. The corresponding correlation coefficients are given in Table 1

fraction monitoring. Alderliesten et al. [17] also compared AlignRT to $3 \mathrm{D}$ CBCT imaging setup errors for DIBH radiotherapy and presented similar results. Calculated LOA were $-0.34-0.48,-0.42-0.39$, and $-0.52-0.23 \mathrm{~cm}$ in the leftright, cranial-caudal, and anterior-posterior directions, respectively. Their results were comparable to the LOA calculated in this study, however Alderliesten et al. used only one longitudinal camera for AlignRT monitoring.

From the LOA determined in our study, it can be concluded that $95 \%$ of the differences in set-up errors

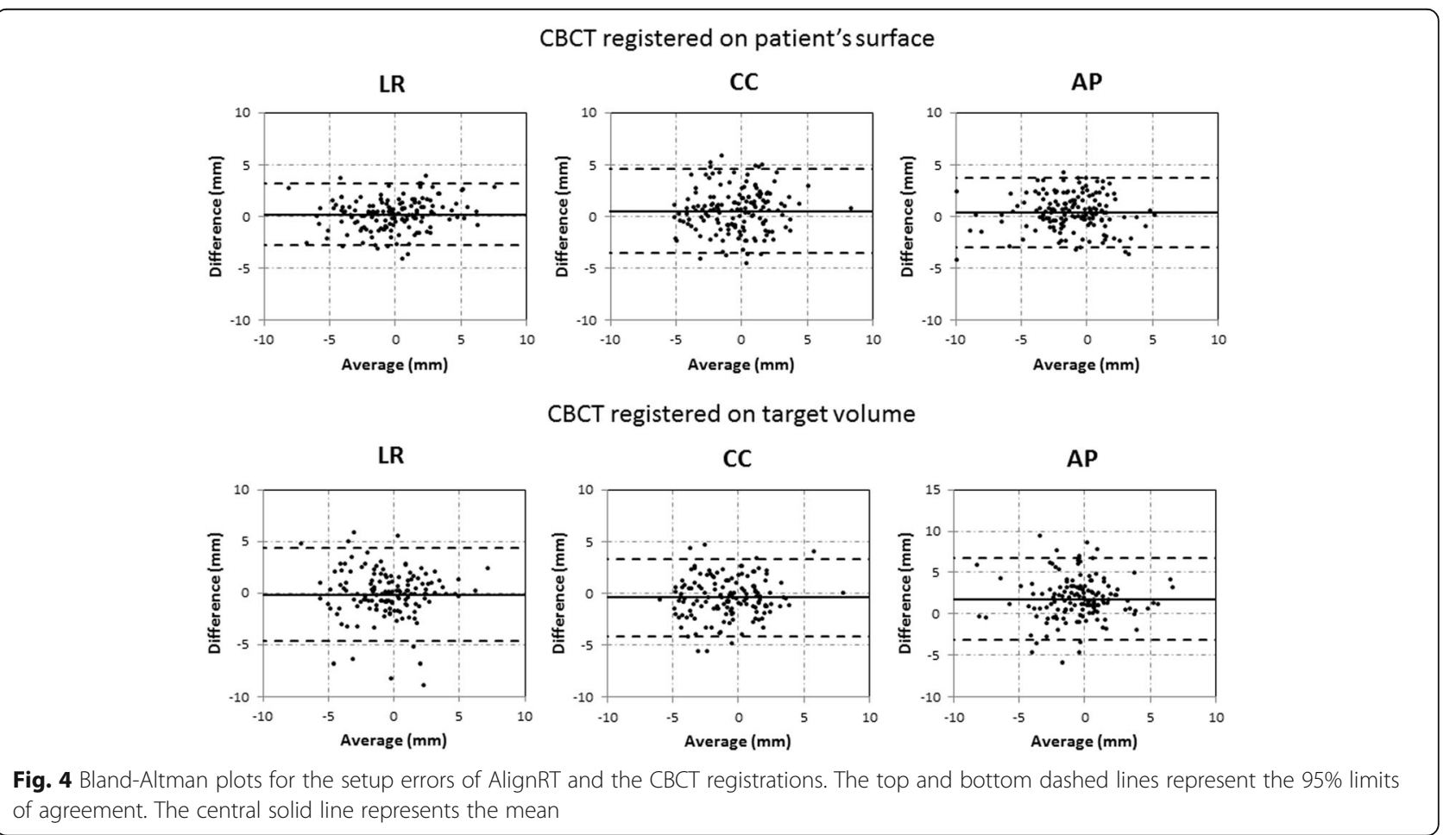


Table 2 Overview of the intra-fraction variability and stability of BHs performed with the $A B C$ system

\begin{tabular}{|c|c|c|c|c|}
\hline & $\mathrm{LR}(\mathrm{mm})$ & $\mathrm{CC}(\mathrm{mm})$ & $\mathrm{AP}(\mathrm{mm})$ & Average \\
\hline \multicolumn{5}{|l|}{ Average ( $\pm 1 S D)$} \\
\hline BH deviation with planning $C T$ & $0.2( \pm 3.1)$ & $-1.0( \pm 2.6)$ & $-1.2( \pm 2.9)$ & $-0.7( \pm 2.9)$ \\
\hline Variability & $2.2( \pm 1.4)$ & $2.8( \pm 1.5)$ & $2.3( \pm 1.3)$ & $2.4( \pm 1.4)$ \\
\hline Stability & $-1.0( \pm 1.3)$ & $2.1( \pm 2.5)$ & $1.5( \pm 2.8)$ & $0.9( \pm 2.2)$ \\
\hline \multicolumn{5}{|l|}{ Maximum } \\
\hline Variability & 7.5 & 12.4 & 6.4 & \\
\hline Stability & -5.8 & 9.6 & 11.7 & \\
\hline
\end{tabular}

BH Breath-hold, LR Left-right, CC Cranial-caudal, AP Anterior-posterior

between AlignRT and the CBCT (surface registered) are within $4.7 \mathrm{~mm}$. Therefore, AlignRT can replace skin markers for initial patient positioning. Other studies have already proven the benefit of AlignRT in patient positioning. Cravo Sá et al. [25] showed that positioning patients with AlignRT is more accurate than when only skin marks are used. Batin et al. [26] showed for postmastectomy patients that positioning with AlignRT after laser alignment resulted in a significant reduction of the residual errors than when positioning with laser alignment alone.

In this study, two different $\mathrm{CBCT}$ registration methods were used. Although slight shading at the surface was visible on the CBCT due to the use of multiple BHs, this did not cause registration inaccuracies. This is because for the $\mathrm{CBCT}$, the resulting image is an average position of the patient, and this is used for registration. The AlignRT data was also analyzed based on an average position over multiple BHs during this study, so the impact of multiple BHs is equivalent for CBCT and SGRT. At first the CBCTs were registered on the patient's surface, as this is the region that AlignRT also uses for registration. Those results can be used to validate the registration accuracy of AlignRT. Average differences between both methods were $\leq 0.6 \mathrm{~mm}$, the $\mathrm{LOA} \leq 4.7 \mathrm{~mm}$ and the $\Sigma$ and $\sigma$ were $\leq 1.4 \mathrm{~mm}$. Therefore, we concluded that AlignRT is acceptable for continuous 3D monitoring, which is not possible with $\mathrm{CBCT}$. The second CBCT registration method was based on the target volume, as this is more relevant to clinical practice. The CBCTs were registered on the thoracic wall and the position errors of the clips and breast contour were maintained within 5 $\mathrm{mm}$. As expected, differences from AlignRT were slightly larger than with the surface-CBCT match. However, the LOA was still within $4.5 \mathrm{~mm}$ for the LR and CC direction. Only for the AP direction the LOA was $6.6 \mathrm{~mm}$. Therefore, we can conclude that $\mathrm{ABC}$ guidance can be used to maintain the target volume position of patients within $4.5 \mathrm{~mm}$ in the LR and CC direction in 95\% of cases and within 6.6 $\mathrm{mm}$ in the AP direction during intra-fraction monitoring.

A higher correlation between AlignRT and the surfaceCBCT data was observed for the first 3 fractions compared to the last 3 fractions ( 0.64 vs 0.55 respectively).
This is due to the formation of lymphedema that causes changes in breast size and shape [27-29]. During the CBCT surface registration, the caudal part of the breast is mainly used for alignment in the CC direction. However, due to the ROI position, AlignRT is mainly looking at the ventral side of the breast, which can differ in shape throughout the treatment. This could explain the lower correlation between AlignRT and the CBCT surface registration in the $\mathrm{CC}$ direction.

The Elekta $\mathrm{ABC}$ was used to guide the $\mathrm{BH}$. This system is spirometer-based and ensures a forced $\mathrm{BH}$. The forced $\mathrm{BH}$ experience is uncomfortable for most patients $[7,30,31]$. The system registers the volume inhaled/exhaled by the patient with high reproducibility [32]. However, only the volume is measured, hence this does not guarantee low variability in thorax/breast positions for $100 \%$ of patients [8]. Moreover, the ABC system is not suitable for claustrophobic/anxious patients, who would benefit from an accurate, SGRT-monitored, voluntary $\mathrm{BH}$ method, such as AlignRT.

In this study, the intra-fraction $\mathrm{BH}$ position variability is on average $2.4 \mathrm{~mm}$ (range $2.2-2.8 \mathrm{~mm}$ ). This result is in agreement with previous studies, where the average variability was within $0.5 \mathrm{~mm}$ [33], $2.2 \mathrm{~mm}$ [34], $1 \mathrm{~mm}$ [6] and $3.4 \mathrm{~mm}$ [35] (calculation of the variability was consistent with this study) and $4 \mathrm{~mm}$ (variability determined as the average standard deviation over $\mathrm{BH}$ positions) [36]. Other studies have also shown low variability in $\mathrm{BH}$ positions with the $\mathrm{ABC}$ system $[37,38]$ and other spirometer based systems $[39,40]$. The stability determined in the current study is on average $0.9 \mathrm{~mm}$ (range $-1.0-2.1 \mathrm{~mm}$ ), which is also in close agreement with Cervino et al. [41] where an average stability of $1.5 \mathrm{~mm}$ (range 0.1-4.2 mm) was determined with GateCT.

Although the average $\mathrm{BH}$ variability is small and the stability is good, outliers persist. Values up to $12.4 \mathrm{~mm}$ for the variability and $11.7 \mathrm{~mm}$ for the stability were observed in this study. This was also noticed by Moran et al. [36] who observed displacements up to $19 \mathrm{~mm}$. Furthermore, errors in stability of $14.1 \mathrm{~mm}$ were observed for one patient caused by escape of air during the $\mathrm{BH}$ through a broken mouthpiece (Fig. 2b). Especially 
relevant for such outliers, SGRT provides additional surface displacement information that is not possible to measure with $\mathrm{CBCT}$ or air-volume $\mathrm{BH}$ systems.

The clinical target volume (CTV) to planning target volume (PTV) margin was $5 \mathrm{~mm}$. In this study, the average intra-fraction deviation of the breast/thorax with respect to the planning CT in any translational direction was larger than $5 \mathrm{~mm}$ in 19 of the 261 treatment fractions (7.3\%), which could potentially result in an under-dosage in those fractions. However, a limited effect on the total CTV dose is expected as those $\mathrm{BH}$ deviations were only observed in a limited number of treatment fractions per patient and a robust planning technique was used. Harron et al. [42] studied the impact on CTV coverage for a systematic $5 \mathrm{~mm}$ shift in all directions for breast patients. The dosimetric effect was less than $5 \%$ on the target volume receiving between the $95-107 \%$ of the prescribed dose. Moreover, Fassi et al. [35] showed only a maximum decrease of $2.1 \%$ on the CTV $\mathrm{D}_{95 \%}$ when applying rotations and translations derived from the $\mathrm{BH}$ variability to the original treatment plan. However, the dose gradients towards the heart and lungs remain sensitive to position variation, which can result in under-dosage to the CTV or increased dose to the heart and lungs. Planning techniques for breast radiotherapy are becoming highly modulated with increasing use of VMAT, IMRT (intensity modulated radiotherapy) or IMPT (intensity modulated proton therapy). By incorporating steeper dose gradients than ever before, low inter- and intrafraction variability and good stability in BHs is becoming more important [43]. Robustness evaluation considering position variation as well as $\mathrm{BH}$ level variations is advisable, to determine the effect on the dose to the CTV.

It is expected that the clinical use of SGRT (AlignRT in this study) for DIBH treatments will result in an improved variability, stability and LOA over $\mathrm{BH}$ guidance based on air-volume. This is because SGRT has a similar surface accuracy to $\mathrm{CBCT}$, can reliably monitor the patient surface during $\mathrm{BH}$ and patients must be within a certain tolerance (normally $3-5 \mathrm{~mm}$ ), hence, large position deviations cannot occur.

\section{Conclusion}

With SGRT, left-sided breast cancer patients can be positioned and monitored to within $5 \mathrm{~mm}$ with respect to reference CBCT data. The SGRT system was able to determine that a low intra-fraction variability and good stability can be achieved for most patients with the airvolume BH system. Additional patient position information is available with SGRT, that cannot be detected with $\mathrm{CBCT}$ or air-volume BH systems.

\section{Abbreviations}

ABC: Active breathing coordinator; AP: Anterior-posterior; BH: Breath-hold CBCT: Cone-beam computed tomography; CC: Craniocaudal; CTV: Clinical target volume; DIBH: Deep-inspiration breath-hold; EPID: Electronic portal imaging device; FB: Free breathing; IMPT: Intensity modulated proton therapy; IMRT: Intensity modulated radiotherapy; LOA: Limits of agreement: LR: Left-right; PTV: Planning target volume; ROI: Region of interest; SD: Standard deviation; SGRT: Surface guided radiotherapy; VMAT: Volumetric modulated arc therapy; WBRT: Whole breast radiotherapy

\section{Acknowledgements}

Not applicable.

\section{Authors' contributions}

$\mathrm{VH}$ : Corresponding author, designed the study, performed analysis of the data, interpreted the data, write the manuscript. CV: Assisted in acquiring all the CBCT and AlignRT data. EB, SB, JL, LM and DB: provided intensive feedback on the manuscript, for multiple versions. MS: Assisted in designing the study, assisted in interpreting the data, provided intensive feedback on the manuscript, for multiple versions. All authors have read and approved the final manuscript.

\section{Funding}

The AlignRT system used in this study was provided by VisionRT Ltd. London, UK, for testing purposes. No financial support was provided for research or any other activities relating to the loan of the system to UMC Groningen. The Department of Radiation Oncology in the UMC Groningen also has Research Agreements with IBA, MIRADA and RaySearch.

\section{Availability of data and materials}

The datasets used and/or analysed during the current study are available from the corresponding author on reasonable request.

\section{Ethics approval and consent to participate}

All patient data were obtained as part of a prospective data registration program within the framework of routine clinical practice. The Dutch Medical Research Involving Human Subjects Act is not applicable to data collection as part of routine clinical practice. Therefore, this study was exempt from the ethical approval requirement by the hospital ethics committee.

\section{Consent for publication}

Not applicable.

\section{Competing interests}

The authors declare that they have no competing interests.

\section{Author details}

${ }^{1}$ Department of Radiation Oncology, University of Groningen, University Medical Center Groningen, Hanzeplein 1, 9713, GZ, Groningen, The Netherlands. ${ }^{2}$ Department of Radiation Oncology, Northwest Clinics, Alkmaar, The Netherlands.

Received: 19 April 2019 Accepted: 27 June 2019

Published online: 11 July 2019

\section{References}

1. Hooning MJ, Botma A, Aleman BMP, Baaijens MHA, Bartelink H, Klijn JGM, et al. Long-term risk of cardiovascular disease in 10-year survivors of breast cancer. J Natl Cancer Inst. 2007;99:365-75.

2. Sripathi LK, Ahlawat P, Simson DK, Khadanga CR, Kamarsu L, Surana SK, et al. Cardiac dose reduction with deep-inspiratory breath hold technique of radiotherapy for left-sided breast cancer. J Med Phys. 2017;42:123-7 Available from: http://www.ncbi.nlm.nih.gov/pmc/articles/PMC5618457/. India: Medknow Publications \& Media Pvt Ltd.

3. Bartlett FR, Colgan RM, Donovan EM, Carr K, Landeg S, Clements N, et al. Voluntary breath-hold technique for reducing heart dose in left breast radiotherapy. J Vis Exp. 2014:1-9 Available from: http:// www.jove.com/video/51578/voluntary-breath-hold-technique-forreducing-heart-dose-left-breast.

4. Yeung R, Conroy L, Long K, Walrath D, Li H, Smith W, et al. Cardiac dose reduction with deep inspiration breath hold for left-sided breast cancer radiotherapy patients with and without regional nodal irradiation. Radiat Oncol; 2015;10:1-6. Available from: https://doi.org/10.1186/s13014-015-0511-8. 
5. Mulliez T, Veldeman L, Speleers B, Mahjoubi K, Remouchamps V, Van Greveling $A$, et al. Heart dose reduction by prone deep inspiration breath hold in left-sided breast irradiation. Radiother Oncol. Elsevier Ireland Ltd; 2015;114:79-84. Available from: https://doi.org/10.1016/j.radonc.2014.11.038

6. Kügele M, Edvardsson A, Berg L, Alkner S, Andersson Ljus C, Ceberg S. Dosimetric effects of intrafractional isocenter variation during deep inspiration breath-hold for breast cancer patients using surface-guided radiotherapy. J Appl Clin Med Phys. 2018;19:25-38.

7. Wong JW, Sharpe MB, Jaffray DA, Kini VR, Robertson JM, Stromberg JS, et al The use of active breathing control $(A B C)$ to reduce margin for breathing motion. Int J Radiat Oncol Biol Phys. 1999;44:911-9.

8. White B, Wuenschel S, Zhao T, Lamb J, Low D. SU-GG-J-90: quantification of the thorax-to-abdomen breathing ratio for breathing motion modeling. Med Phys. 2010;37:3166.

9. Goyal S, Kataria T. Image guidance in radiation therapy: techniques and applications. Radiol Res Pract. 2014;2014:1-10 Available from: http://www. hindawi.com/journals/rrp/2014/705604/. Hindawi Publishing Corporation.

10. Hoisak JDP, Pawlicki T. The role of optical surface imaging Systems in Radiation Therapy. Semin Radiat Oncol. Elsevier; 2018;28:185-193. Available from: https://doi.org/10.1016/j.semradonc.2018.02.003

11. Pan H, Cerviño LI, Pawlicki T, Jiang SB, Alksne J, Detorie N, et al. Frameless, real-time, surface imaging-guided RadiosurgeryClinical outcomes for brain metastases. Neurosurgery 2012;71:844-852. Available from: https://doi.org/ 10.1227/NEU.0b013e3182647ad5.

12. Bartlett FR, Colgan RM, Carr K, Donovan EM, McNair HA, Locke I, et al. The UK HeartSpare Study: Randomised evaluation of voluntary deep-inspiratory breathhold in women undergoing breast radiotherapy. Radiother Oncol; 2013;108:242_ 247. Available from: https://doi.org/10.1016/j.radonc.2013.04.021. Elsevier Ireland Ltd

13. Hamid S, Ceberg S, Kügele M, Bergh L, Gunnlaugsson A. A comparison of surface based and laser based setup for rectal cancer patients in radiotherapy. 2017 [cited 2018 Jul 6]. Available from: http://lup.lub.lu.se/ student-papers/record/8927723

14. Crop F, Pasquier D, Baczkiewic A, Doré J, Bequet L, Steux E, et al. Surface imaging, laser positioning or volumetric imaging for breast cancer with nodal involvement treated by helical TomoTherapy. J Appl Clin Med Phys. 2016;17:200-11.

15. Fayad H, Wentz T, Pan T, Visvikis D. SU-E-J-148: correlation of respiratory motion between external patient surface and internal anatomical landmarks. Med Phys. 2011;38:3477.

16. Jensen CA, Roa AMA, Johansen M, Lund JÅ, Frengen J. Robustness of VMAT and 3DCRT plans toward setup errors in radiation therapy of locally advanced left-sided breast cancer with DIBH. Phys Medica. 2018;45:198-204.

17. Alderliesten T, Sonke JJ, Betgen A, Honnef J, Van Vliet-Vroegindeweij C, Remeijer P. Accuracy evaluation of a 3-dimensional surface imaging system for guidance in deep-inspiration breath-hold radiation therapy. Int J Radiat Oncol Biol Phys. Elsevier Inc:; 2013;85:536-542. Available from: https://doi.org/10.1016/j.jjrobp.2012.04.004

18. Bland JM, Altman DG. Statistical methods for assessing agreement between two methods of clinical measurement. Lancet. 1986:327:307-10 Available from: http://www.sciencedirect.com/science/article/pii/S0140673686908378.

19. Shah AP, Dvorak T, Curry MS, Buchholz DJ, Meeks SL. Clinical evaluation of interfractional variations for whole breast radiotherapy using 3-dimensional surface imaging. Pract Radiat Oncol. 2013;3:16-25 Available from: http:// www.sciencedirect.com/science/article/pii/S1879850012000409.

20. Krengli M, Gaiano S, Mones E, Ballarè A, Beldì D, Bolchini C, et al. Reproducibility of patient setup by surface image registration system in conformal radiotherapy of prostate cancer. Radiat Oncol. 2009:4:1-10.

21. Bartoncini S, Fiandra C, Ruo Redda MG, Allis S, Munoz F, Ricardi U. Target registration errors with surface imaging system in conformal radiotherapy for prostate cancer: study on 19 patients. Radiol Med. 2012;117:1419-28. Available from. https://doi.org/10.1007/s11547-012-0823-9.

22. Padilla L, Kang $H$, Washington $M$, Hasan $Y$, Chmura SJ, Al-Hallaq H. Assessment of interfractional variation of the breast surface following conventional patient positioning for whole-breast radiotherapy. J Appl Clin Med Phys. 2014;15:177-89.

23. Deantonio L, Masini L, Loi G, Gambaro G, Bolchini C, Krengli M. Detection of setup uncertainties with $3 D$ surface registration system for conformal radiotherapy of breast cancer. Rep Pract Oncol Radiother. Wielkopolskie Centrum Onkologii; 2011;16:77-81. Available from: https:// doi.org/10.1016/j.rpor.2011.02.003.

24. Wolken T. Comparing the accuracy of AlignRT and exactrac at intrafractional time points during cranial stereotactic radiosurgery and stereotactic radiotherapy treatments; 2018.
25. Cravo Sá A, Fermento A, Neves D, Ferreira S, Silva T, Marques Coelho C, et al. Radiotherapy setup displacements in breast cancer patients: 3D surface imaging experience. Rep Pract Oncol Radiother. Greater Poland Cancer Centre; 2018;23:61-67. Available from: https://doi.org/10.1016/j.rpor.2017.12.007.

26. Batin E, Depauw N, MacDonald S, Lu H-M. Can surface imaging improve the patient setup for proton postmastectomy chest wall irradiation? Pract Radiat Oncol. 2016;6:e235-41 Available from: http://www.sciencedirect.com/ science/article/pii/S1879850016000503.

27. Adriaenssens N, Belsack D, Buyl R, Ruggiero L, Breucq C, De Mey J, et al. Ultrasound elastography as an objective diagnostic measurement tool for lymphoedema of the treated breast in breast cancer patients following breast conserving surgery and radiotherapy. Radiol Oncol. 2012;46:284-95.

28. Adriaenssens $\mathrm{N}$, Verbelen $\mathrm{H}$, Lievens $\mathrm{P}$, Lamote J. Lymphedema of the operated and irradiated breast in breast cancer patients following breast conserving surgery and radiotherapy. Lymphology. 2012;45:154-64 Available from: http://www.ncbi. nlm.nih.gov/pubmed/23700762

29. Toledano A, Garaud P, Serin D, Fourquet A, Bosset JF, Breteau N, et al. Concurrent administration of adjuvant chemotherapy and radiotherapy after breastconserving surgery enhances late toxicities: Long-term results of the ARCOSEIN multicenter randomized study. Int J Radiat Oncol Biol Phys. 2006;65:324-32.

30. Dawson LA, Brock KK, Kazanjian S, Fitch D, McGinn CJ, Lawrence TS, et al. The reproducibility of organ position using active breathing control $(A B C)$ during liver radiotherapy. Int J Radiat Oncol Biol Phys. 2001;51:1410-21.

31. Garcia R, Oozeer R, Le Thanh H, Chastel D, Doyen JC, Chauvet B, et al. Radiothérapie des du poumon: Le blocage en inspiration sous contrôle spirométrique. Cancer/Radiotherapie. 2002;6:30-8.

32. Kaza E, Dunlop A, Panek R, Collins DJ, Orton M, Symonds-Tayler R, et al. Lung volume reproducibility under $A B C$ control and self-sustained breathholding. J Appl Clin Med Phys. 2017;18:154-62.

33. Betgen A, Alderliesten T, Sonke JJ, Van Vliet-Vroegindeweij C, Bartelink H, Remeijer P. Assessment of set-up variability during deep inspiration breath hold radiotherapy for breast cancer patients by 3D-surface imaging. Radiother Oncol. Elsevier Ireland Ltd; 2013;106:225-230. Available from: https://doi.org/10.1016/j. radonc.2012.12.016

34. Xiao A, Crosby J, Malin M, Kang H, Washington M, Hasan Y, et al. Single-institution report of setup margins of voluntary deep-inspiration breath-hold $(\mathrm{DIBH})$ whole breast radiotherapy implemented with real-time surface imaging. J Appl Clin Med Phys. 2018;19:205-13.

35. Fassi A, Ivaldi GB, Meaglia I, Porcu P, Tabarelli de Fatis P, Liotta M, et al. Reproducibility of the external surface position in left-breast $\mathrm{DIBH}$ radiotherapy with spirometer-based monitoring. J Appl Clin Med Phys. 2014;15:4909.

36. Moran JM, Balter JM, Ben-David MA, Marsh RB, Van Herk M, Pierce $\sqcup$. Short-term displacement and reproducibility of the breast and nodal targets under active breathing control. Int J Radiat Oncol Biol Phys. 2007:68:541-6.

37. Mittauer KE, Deraniyagala R, Li JG, Lu B, Liu C, Samant SS, et al. Monitoring ABC-assisted deep inspiration breath hold for left-sided breast radiotherapy with an optical tracking system. Med Phys. 2015;42:134-43.

38. Remouchamps VM, Letts N, Vicini FA, Sharpe MB, Kestin LL, Chen PY, et al. Initial clinical experience with moderate deep-inspiration breath hold using an active breathing control device in the treatment of patients with left-sided breast cancer using external beam radiation therapy. Int J Radiat Oncol Biol Phys. 2003;56:704-15.

39. Rosenzweig KE, Hanley J, Mah D, Mageras GS, Hunt M, Toner S, et al. The deep inspiration breath-hold technique in the treatment of inoperable non-small-cell lung cancer. Int J Radiat Oncol Biol Phys. 2000:48:81-7.

40. Mah D, Hanley J, Rosenzweig KE, Yorke E, Braban L, Ling CC, et al. Technical aspects of the deep inspiration breath-hold technique in the treatment of thoracic cancer Int J Radiat Oncol Biol Phys. 2000:48:1175-85.

41. Cerviño LI, Gupta S, Rose MA, Yashar C, Jiang SB. Using surface imaging and visual coaching to improve the reproducibility and stability of deep-inspiration breath hold for left-breast-cancer radiotherapy. Phys Med Biol. 2009;54:6853-65.

42. Harron EC, McCallum HM, Lambert EL, Lee D, Lambert GD. Dosimetric effects of setup uncertainties on breast treatment delivery. Med Dosim. 2008;33:293-8.

43. Guadagnolo BA, Liu CC, Cormier JN, Du XL. Evaluation of trends in the use of intensity-modulated radiotherapy for head and neck cancer from 2000 through 2005: socioeconomic disparity and geographic variation in a large population-based cohort. Cancer. 2010;116:3505-12.

\section{Publisher's Note}

Springer Nature remains neutral with regard to jurisdictional claims in published maps and institutional affiliations. 